\title{
INNOVACIONES EN LA RIMA: POESÍA Y RAP
}

\begin{abstract}
Clara I. Martínez Cantón
Resumen: El uso de la rima ha decrecido considerablemente en la poesía actual en español por varias razones, entre las que destaca la transición de la oralidad a una cultura escrita. En realidad, gracias a la cultura oral la rima no se ha perdido en las letras de canciones, sino más bien todo lo contrario. En algunos estilos de música su uso se ha reforzado y se ha comenzado a experimentar con sus vastas posibilidades. En este artículo analizaremos estos experimentos que prueban que hay todavía mucho que descubrir en este campo, y que la rima no es un elemento tan limitado como podía parecer. Nos centraremos únicamente en un estilo, el rap, y en un grupo, Violadores del Verso. Trataremos, asimismo, de relacionar y comparar estas innovaciones de la rima que se utilizan en la música con las utilizadas en la poesía en lengua española actual.
\end{abstract}

Palabras clave: Rima, rap, experimentación, innovación, letras de canciones, poesía actual.

Abstract: The use of rhyme in the contemporary Hispanic poetry has notoriously decreased for several reasons amongst which the most remarkable is probably the transition from oral culture to written culture. Actually, it is thanks to oral culture that rhyme has not been lost in lyrics, rather the opposite. In some music styles it has been reinforced and the experimentation of its endless possibilities has begun. This paper will analyse such 
experiments that prove there is indeed a lot yet to discover in this field, and that rhyme is not as restricted as it may seem. We will focus on just one music style, rap, and just one group, Violadores del Verso. We will try, as well, to relate and compare these innovations on rhyme used in music with the ones used in contemporary Hispanic poetry.

Key words: Rhyme, rap, experimentation, innovation, lyrics, contemporary poetry. 


\section{INTRODUCCIÓN}

UNQUE resulte sorprendente, las investigaciones y es-
tudios de métrica españoles e hispanoamericanos se
ocupan rara vez del campo de las letras de canciones y de su posible comparación con la métrica de la poesía. Esto puede venir provocado por las diferencias que podemos apreciar entre "poesía" y "canción", que básicamente se podrían resumir en una que engloba todas las demás: la poesía está hecha para ser leída o recitada, mientras que las letras de las canciones van unidas a una melodía, que determina en cierta manera su medida, centros de intensidad, etc.

Este hecho repercute decisivamente en ciertos elementos métricos. La medida silábica puede variar mucho de unos versos a otros, ya que una misma sílaba puede alargarse más o menos, es decir, entra el factor "duración de la sílaba", que en la poesía en lengua española no tiene relevancia. Por otra parte, también la pausa se ve afectada, pues en muchos casos, una de características más reconocibles de la poesía, es decir, la existencia de versos separados por la llamada pausa versal, no es perceptible, ya que la música crea en ciertos casos un continuum que hace difícil y dudoso el separar en distintas líneas versales el discurso. El acento pierde importancia en el ritmo, que viene marcado por la música. La rima, sin embargo, mantiene, en general, un papel parecido al que tiene en la poesía: su utilización, aunque común, no es obligatoria, y cuando aparece, en muchas ocasiones sirve para marcar en el texto la existencia de distintas líneas que podríamos llamar versales.

A pesar de todo, los parecidos entre las letras de las canciones y los poemas son evidentes. De hecho, como es sabido, 
su origen es común, y sólo con el paso del tiempo se diferenciarían las dos artes. Siempre han existido, no obstante, formas mixtas, y algunos tipos de poesía popular, como los romances, han sido cantados sobre todo en las zonas rurales hasta la actualidad con un tipo de música repetitiva, tradicionalmente llamada "tonada"l.

En la actualidad, sin embargo, parece que poesía y música se encuentran separadas por una línea bastante clara: la poesía se lee y la música (y sus letras) se escuchan. Esto no es siempre y obligatoriamente así, como sabemos, y al igual que existen recitales de poesía, existen libros con letras de canciones, pero es evidente que el modo de recepción actual más común y natural de cada una es el dicho anteriormente. Esto sucede sobre todo a partir de la llamada "crisis de la oralidad", que cambia el modo de recepción de la poesía y con ello repercute de forma decisiva en ella. La forma, los elementos rítmicos, sonoros y visuales que dan cuerpo al verso varían sustancialmente; veamos por ejemplo la ligazón entre verso libre y lectura como modo de recepción de la poesía:

El verso libre nace con lo que he llamado crisis de la oralidad. En efecto, en el poema tradicional, el oyente necesitaba una serie de "marcas" o puntos de apoyo, como la rima o la pausa versal, además del ritmo y el metro, para fijar el estatuto poético del texto que escuchaba y para, ocasionalmente, memorizarlo. El lector individual y solitario dispone de las marcas gráficas, por lo que el poeta puede prescindir -y el poeta moderno prescindió, de hecho- de alguna o algunas de las marcas de la poesía oral tradicional, dando lugar al nacimiento del verso libre 2 .

Esto nos lleva al punto clave de nuestro trabajo: mientras que en la poesía la rima ha experimentado una recesión en $\mathrm{su}^{\mathrm{uso}^{3}}$, en las letras de canciones la rima ha persistido en

' El DRAE define este término de la siguiente manera: "Tonada: 1. f. Composición métrica para cantarse. 2. f. Música de esta canción”. Real Academia Española de la Lengua: Diccionario de la lengua española. Http://buscon.rae. es/draeI/html/cabecera.htm, 2001.

2 MARTÍNEZ FERNÁNDEZ, José Enrique: El fragmentarismo poético contemporáneo. León: Universidad de León, 1996, pp. 51-52.

3 MARTÍN CASAMITJANA liga este fenómeno a "las novedades tipográficas" y data su comienzo en los años treinta del pasado siglo (MARTÍN CASAMI- 
sus diversas formas, e incluso ha comenzado a innovarse. La introducción de novedades en este elemento es algo muy llamativo porque parece que era el componente métrico con el que menos se había experimentado y cuyos límites parecían más claros.

En poesía la rima ha sido siempre un elemento muy polémico, pues ha sufrido desprestigio en algunas épocas y autores, acusada de grandes defectos, mientras que en otros casos se ha tenido en cuenta su valor expresivo. El vicio mayor que se le ha atribuido a la rima es que condiciona el contenido, es decir, el uso de la rima sin sentido, "la rima como impulso que se impone al poeta y lo guía por donde su propia voluntad no lo habría llevado"4. También se le achaca una sonoridad que puede resultar excesiva para el oído, llegando a perjudicar la recepción del texto poético. Como virtudes cabe destacar que la rima es un recurso muy eficaz para la memorización de un poema, pero sobre todo, que la rima es capaz de crear asociaciones semánticas muy ricas y sugerentes. Así, “el buen poeta será el que se muestra capaz de vencer esas dificultades y satisfacer las exigencias de la rima mediante su dominio del idioma y de la técnica de la versificación, sin que ello le desvíe de su propósito" 5 . La rima, por tanto, es un mecanismo eficaz en múltiples aspectos de la lengua poética, y en palabras de Mónica Güell proporciona una lectura "simultáneamente horizontal y vertical, paradigmática y sintagmática, de la cual surgen alianzas léxicas o semánticas previsibles, convenidas, siguiendo los códigos poéticos vigentes, o al contrario, parejas o tríos sorprendentes, no convencionales"6.

La rima ha acompañado siempre a la canción y la poesía popular, y ha sido precisamente la poesía más culta la que

TJANA, Rosa $\mathrm{M}^{\mathrm{a}}$ : El humor en la poesía española de vanguardia. Madrid: Gredos, 1996, p. 58).

4 FRAU, Juan: "Teorías y polémicas sobre la rima en el Renacimiento inglés". Rhythmica. Revista española de métrica comparada, 2008, V-VI, p. 61.

5 Ibid., p. 83.

6 GÜELL, Mónica: "La creatividad de las rimas en las décimas y letrillas de Góngora”, en Anthony J. Close, Sandra María Fernández Vales (coords.), Edad de oro cantabrigen. Actas del VII Congreso de la Asociación Internacional de Hispanistas del Siglo de Oro. Asociación Internacional del Siglo de Oro, 2006, p. 331 . 
con frecuencia se ha desligado de este elemento. Trataremos de dar en este trabajo una visión de cómo la rima todavía tiene mucho que ofrecer y nuevos campos por explorar, tanto en música como en poesía, y para ello nos centraremos en las que Domínguez Caparrós ha denominado "rimas extrasistemáticas" y que ha definido como "casos y experimentos que se sitúan en las fronteras de la definición normativa del sistema de la rima, y que destacan el papel rítmico que puede desempeñar el amplio campo retórico de la eufonía o de la paranomasia" 7 .

La organización que seguiremos para dar cuenta de estos cambios y avances será la siguiente. Ya que no podemos reflejar todas las novedades que han aparecido en todos los géneros musicales respecto a la rima y para establecer unos límites en un campo que en otro caso sobrepasaría las posibilidades de este trabajo, nos centraremos específicamente en un estilo musical concreto y en el grupo que consideramos más representativos en el ámbito hispánico y que más ha innovado en este aspecto. Hemos seleccionado para ello a Violadores del Verso, grupo que realiza hip-hop en español. Trataremos por último de relacionar y comparar el uso de estas innovaciones en la rima en este grupo y género musical con las practicadas en la poesía actual española.

\section{Hip-HOP. Violadores del VERSO}

Uno de los pocos estudios dentro del ámbito académico que he hallado sobre este tema es el titulado "El resurgir de la rima: los poetas románicos del rap", de Santos Unamuno, y comienza de esta forma que creo interesante reproducir y comentar:

Hablar o escribir de manifestaciones culturales como el hip hop o el rap en ámbito académico y universitario (sobre todo en Europa)

7 DOMÍNGUEZ CAPARRÓS, José: "La rima: entre el ritmo y la eufonía", en Estudios de métrica. Madrid: Universidad Nacional de Educación a Distancia, 1999, p. 179. 
entraña cierta dificultad, pues se corre el riesgo de ser acusado de sustituir a Dante o Garcilaso por adalides de la cultura de masa en nombre de un pretendido Resentimiento y de fomentar esa "balcanización de los estudios literarios" tan temida y aborrecida por el clasicista Harold Bloom [...] Abogar por la apertura y la remodelación de los cánones, abordarlos desde un punto de vista histórico, sustituyendo la verticalidad jerárquica por geometrías más fluidas, no significa forzosamente sustituir a los clásicos sino dar derecho de ciudadanía a otras voces $^{8}$.

También Pujante Cascales, en un artículo dedicado precisamente al estudio de las figuras retóricas del mismo grupo del que aquí nos ocuparemos, Violadores del Verso, comienza de una manera similar: "Género musical reciente y altamente subversivo, el rap no ha encontrado fácil acomodo, al menos en nuestro país, en los estudios académicos" ". Nos enfrentamos entonces a un género poco estudiado desde el punto de vista académico y más aún desde el punto de vista filológico, que, sin embargo, no cabe duda de que puede resultar muy interesante, pues comparte ciertos elementos con la literatura y la poesía que pueden enriquecer nuestros estudios.

El rap es un género musical que, aunque entró tarde en España, ha ido creciendo en popularidad paulatinamente. Pujante señala como fecha clave 1994, con la publicación de Madrid, Zona Bruta, primer LP de El Club de los Poetas Violentos. Precisamente un miembro de este grupo pionero, Jota Mayúscula, DJ, colaboró ${ }^{10}$ también en uno de los primeros trabajos del grupo del que vamos a hablar, en "Genios" (1999).

Pero dejémonos ya de prolegómenos y comencemos presentando a Violadores del Verso o, como también se les conoce,

8 SANTOS UNAMUNO, Enrique: "El resurgir de la rima: los poetas románicos del rap", en Antonella Cancellier y Renata Londero (coords.), Atti del XIX Convegno [Associazione ispanisti italiani]: Roma, 16-18 settembre 1999, vol. 2, 2001, p. 235.

9 PUJANTE CASCALES, Basilio: "La retórica del rap. Análisis de las figuras retóricas en las letras de Violadores del Verso". Tonos digital: Revista electrónica de estudios filológicos, 2009, n. 17.

10 Jota Mayúscula ha colaborado también con muchos otros grupos y MCs punteros en el panorama del rap en España: SFDK en "Siempre fuertes" (1999) o La Mala Rodríguez en "Yo marco el minuto" (1999), "Lujo Ibérico" (2000) y "Alevosía” (2003). 
Doble V. Este grupo de rap zaragozano, que como hemos dicho comienza su andadura a finales de los noventa, está compuesto por Hate (también llamado Sho Hai) (Sergio Rodríguez, MC), Kase-O (también conocido como Javat) (Javier Ibarra, MC), Lírico (David Gilaberte, MC) y R de Rumba (Rubén Cuevas, DJ y productor) y, antiguamente, Brutal (Sergio Ibarra, DJ y productor). Miguel Ángel Sutil, en un libro dedicado al grupo, afirma que a partir de la publicación en 2001 de su disco Vicios y virtudes "por ventas, conciertos y repercusión [Violadores] se convierte en el grupo de referencia para chequear el estado de salud del género en España, y sus conciertos cada vez aglutinan a más público" ${ }^{11}$. Dado que dejaremos a un lado la música y nos centraremos en las letras de las canciones, nos interesan especialmente los llamados MCs, maestros de ceremonias o vocalistas, que son los que crean las letras. En Violadores del Verso es Javier Ibarra, más conocido como Kase-O el que utiliza rimas más arriesgadas, y por lo tanto, el autor de la mayoría de las letras que comentaremos.

Conviene hacer ciertas aclaraciones antes de comentar las innovaciones en el campo de la rima, ya que nos ayudarán a entender la forma de concebir ciertos fenómenos que priman en este género en temas como la creación de letras, la propia rima, etc. Respecto a la relación de su obra con la literatura Santos Unamuno afirma que:

Una cosa resulta evidente: los autores de canciones rap atribuyen a sus creaciones una intención y un valor declaradamente artístico, seguros de hallarse inmersos en el devenir de los géneros poéticos. Esta autoconciencia es bien visible en el uso constante de palabras como "métrica", "lírica", "verso", "poesía" y, sobre todo, "rima", la palabra clave del universo rap ${ }^{12}$.

En las letras de Violadores del Verso encontramos múltiples alusiones a este hecho: "Calculo mis métricas pensando

11 SUTIL, Miguel Ángel: Los hijos secretos del funk. Zaragoza: Zona de obras, 2008, p. 67.

12 SANTOS UNAMUNO: "El resurgir de la rima: los poetas románicos del rap", cit., p. 239. 
en la mejor carambola" ("Filosofía y letras", Vivir para contarlo), "podréis odiar al poema pero no al poeta" ("Modestia aparte", Vicios y virtudes), e incluso referencias a la tradición literaria: "bebí del biberón de Cicerón y de Virgilio, hoy soy poeta con emoción a domicilio" etc. Esto nos lleva a una cuestión fundamental, que es la de la importancia de la rima en las letras del rap. La rima se erige dentro de este género como un elemento fundamental; escribe Pujante en el rap la rima se convierte prácticamente en una obsesión: "El MC debe buscar siempre la ecuación perfecta para sus letras en forma de rima sorprendente y exacta que deje boquiabiertos a los demás raperos y al público"13. La rima se erige como elemento de apoyo y como generador de nuevas asociaciones sorprendentes, pero además, en el rap, se fomenta de forma especial la sonoridad. Esa sonoridad que, dicho sea de paso, es la otra clave de este estilo, que se basa en una especie de salmodia rítmica, conocida como "flow", en el que destacan principalmente las pausas y los silencios ${ }^{14}$, que hacen más perceptible asimismo la rima.

Llegados a este punto podemos comenzar ya con el análisis de la rima en las canciones de este grupo. Nos centraremos en aquellas que pueden resultar más sorprendentes e innovadoras desde el punto de vista de la tradición literaria. Como acabamos de decir, la "eufonía" es algo especialmente buscado en las letras y rimas de los raperos, pero además la rima debe ser original, sorprender. Según dice Pujante Cascales: "Desde el punto de vista fonológico el ingenio se demuestra mediante la eufonía del rapeo, el "flow" en el argot del hiphop, y mediante la elaboración y originalidad de la rima"15.

13 PUJANTE CASCALES: "La retórica del rap. Análisis de las figuras retóricas en las letras de Violadores del Verso", cit.

14 En el libro de SUTIL se plantea a Violadores la siguiente pregunta, que resulta bastante esclarecedora, pues da idea de la importancia del ritmo en el recitado de las letras: "Yo creo que en el fondo no hay tanta diferencia entre un solo de jazz y hacer un rap. Rapear puede llegar a ser como hacer un solo, tienes que hacerlo fluido y llamativo para que sea bueno. Incluso creo que se podría intentar sacar con un instrumento la melodía de una frase rapeada. Hay ejemplos de gente que lo ha intentado", para terminar sentenciando "el rap es el juego de los silencios y las pausas". (SUTIL: Los hijos secretos del funk, cit., pp. 73-74.)

15 PUJANTE CASCALES: "La retórica del rap. Análisis de las figuras retóricas 
Por ello este género musical experimenta constantemente con este elemento.

En general, podemos decir que la rima se utiliza en Violadores del Verso de una manera muy libre, tanto en su forma consonante como en la asonante, aunque hay una marcada tendencia al primero de estos tipos. Pujante describe la estructura métrica más común en estas letras como "largas tiradas de versos con distintas rimas y en algunos casos estribillos que se repiten varias veces durante la canción" ${ }^{16}$. Destaca además la importancia de la rima interna, muy habitual, y que tiende a marcar asimismo un ritmo.

Uno de los cambios más comunes respecto a la rima tradicional que encontramos en este grupo es el cambio de acento en las palabras ${ }^{17}$, que da lugar a rimas que de otro modo serían imposibles. Veamos algunos ejemplos de la canción "Modestia aparte":

Colega , calma tu cólera o te dolerá, mi Rap , llegó a tu bóveda y no se moverá, No me vendí, que va!, me defendí, no creí en tí ni en tí por eso estoy aquí en D\&D Algunas tías sonríen, pues saben quien rapea bien, nací en el infierno así que no se fíen A cien mc's metí en crisis ¿a mí? sí...¿¿tú? sí... ni en Midi ni en DVD ${ }^{18}$

\footnotetext{
en las letras de Violadores del Verso", cit.

16 Ibid.

17 Este recurso es conocido también como "éctasis", y es definido por DEVOTO de la siguiente manera: "«Unico, en lugar de único por la fuerza del consonante y por deliberada jocosidad. Góngora escribió... Napoles... Francía. Y Cervantes, en el Viaje del Parnaso: "poeta ilustre, o al menos, magnifico". A esto llamaban éctasis los antiguos retóricos», Rodríguez Marín, 21.000 refranes, 355. La voz se usa en Lebrija, de donde la toma MZAmador, 534”. DEVOTO, Daniel: Para un vocabulario de la rima española. Paris: Klincksieck, 1995, p. 83.

18 De aquí en adelante señalizaremos en negrita las palabras rimadas que queremos destacar. En el caso de que la rima provoque un desplazamiento de acento será comentado más adelante, pero no hemos considerado adecuado marcar gráficamente con una tilde estos acentos prosódicos, ya que, como veremos en este estudio, lo que opera no es un simple cambio de sílaba del acento, sino que muchas veces es una manera diferente de pronunciar, marcando cada sílaba, singularizándolas.
} 
En este breve fragmento encontramos muchos recursos destinados a potenciar la rima. Comencemos por los dos primeros versos. En ellos vemos cinco palabras trisílabas que comparten todas las vocales: colega, cólera, dolerá, bóveda, moverá. De ellas hay dos agudas, una llana y dos esdrújulas. Lo que se opera, sin embargo, no es un simple cambio de acento para que todas ellas rimen, sino que se trata de que en el recitado se enfatizan cada una de las sílabas, de modo que el acento prosódico se difumina y crea la sensación de rima, ayudada además por la existencia de otras figuras de eufonía como la aliteración.

Caso distinto es el de los versos que continúan, en los que, sin embargo, se acentúa claramente la letra "i", lo cual no provoca ningún cambio en la dicción hasta el verso quinto de los aquí reproducidos, en el que se destaca provocando un efecto que, aventurándonos un poco, podríamos llamar de rima entre la "i" de "tías" y "sonríen". Pero lo más sorprendente está aún por llegar y es la rima entre "creí en", "aquí en", "D\&", "sonríen", “quien”, "bien”, "nací en”, "fíen”, "cien” y "metí en". En realidad el apoyo, la mayor intensidad en la pronunciación recae siempre en la citada vocal "i”, por lo que el final de la que podemos llamar tirada con la misma rima termina rimando solamente "mí", "sí", "Midi" y "DVD", al igual que al principio comenzó con "vendí" o "defendí". Que la intensidad recaiga en esa vocal requiere un cambio claramente perceptible del lugar de la acentuación normal en la palabra en términos como "quien", "bien" o "cien", que pasarían a ser bisílabas, realizándose un hiato entre las dos vocales que las forman. También encontramos una pronunciación especial de los vocablos que, aun tratándose de dos palabras diferentes, actúan como una sola para formar la rima: "creíen" (creí en), "aquíen"( aquí en), "nacíen" (nací en), "metíen" (metí en), o incluso en inglés "D\&". Antes hablábamos de la rima interna y aquí observamos claramente la importancia de la misma ${ }^{19}$, que en esta parte de la canción actúa sin ninguna duda como

19 Este rasgo tiene más relevancia en unos MC's que en otros. La letra del citado fragmento pertenece a Kase.O, que practica con mucha frecuencia la rima interna. 
marca rítmica, forzando incluso los acentos prosódicos. Así la "í" coincide con el tiempo fuerte del compás resaltándose y consiguiéndose una unión entre ritmo musical, acentual y de rima.

El desplazamiento del acento prosódico natural de la palabra es algo muy normal en las letras de este grupo y podríamos poner gran cantidad de ejemplos:

vivir la vida como en una película, eyacula esdrújulas $\mathrm{J}$ mayúscula (Ballantines)

voy en la misma dirección que fluyen los ríos, acepto desafíos, otras veces me cabreo con Dios

(Filosofía y letras)

Co, estas manos que te palpan

luego irán al pan de mi mejor fan (Javat y Kamel)

sigo esperando el latido del telefono

todo mi honor en torno a un tono que no sono

(Ninguna chavala tiene dueño)

En muchos de los casos, como estos dos anteriores, se resalta cada una de las sílabas, por lo que lo más sorprendente al oído es la separación en dos sílabas de un diptongo, como en el caso de "Dios". En el último verso aquí recogido es significativo que además de la rima se mezclan otros recursos fónicos, como la paranomasia, la aliteración o la armonía vocálica. Pero encontramos que se resalta asimismo una rima interna muy clara: "honor" y "tor-(no)", en la que esta segunda palabra se realiza marcando una separación clara de sílabas. En realidad, esta forma de hacer rimar palabras se podría definir con el término de "rima potencial", acuñado por Luis Ángel Casas, un poeta cubano, que el estudioso Luis Mario recoge y explica así:

La rima potencial se basa, pues, en el concepto de igualdad silábica pura o semejanza silábica pura, según los casos, pero siempre con independencia del acento. Dicho más académicamente, este tipo de rima es la igualdad o semejanza entre la sílaba tónica y la postónica o protónica; entre la postónica y la protónica y entre las protónicas, 
en palabras de cualquier tipo de acentuación; y, también entre las protónicas no equidistantes de la tónica, cuando se trata de palabras esdrújulas o sobresdrújulas ${ }^{20}$.

Hay otros casos en los que existe realmente un claro cambio en el lugar del acento, para conseguir la rima final de verso:

escribo lo que siento y lo que veo también, que no sé criticar sin insultar también, que yo puedo hacer que las cosas cambien,

(Javat y Kamel)

Vengo con rimas kilométricas, no competirás, Luego me dirás: "Jodo cabrón como deliras," Toma medidas, cura tu rap de estas heridas, Chupa de mi flash, bebe bebidas energéticas,

(Pura droga sin cortar)

En otros casos más que de desplazamiento del acento tendríamos que hablar del uso de palabras que terminan en la misma sílaba, aunque ésta sea átona. No se produce tampoco una rima en caída, sino que simplemente, al repetirse la misma sílaba encuentra una resonancia. El concepto más cercano que he encontrado es quizás el de "anafonema", que recoge Devoto en su Vocabulario y define así: "Balbín Lucas (según DzCap 2) designa así la repetición mantenida de la vocal átona en versos consecutivos (la a terminal de las rimas de Darío en el ejmplo de DzCap: lira- griega-inspira-navega-sagradaseda-rosada-Leda); véase «rima de tirada» al final del artículo rima" 21 , pero como vemos no es exacto, ya que en los casos que vamos a ver se repite toda la sílaba, que puede ser átona o no, y no solamente una vocal:

He rimado miles de palabras y aun quiero más,

Sobre el ritmo sabes que les enseñé mil formas

(Pura droga sin cortar)

Pero no te hagas el tonto, co, no culpes al público,

${ }_{20}$ MARIO, Luis: Ciencia y arte del verso castellano. Miami: Ediciones Universal, 1991, p. 151.

21 DEVOTO, Daniel: Para un vocabulario de la rima española, cit., p. 239. 
Huele mal y es tu estilo culofónico (Pura droga sin cortar)

Aunque no podemos hablar propiamente de rima por tmesis o encabalgamiento léxico, sí cabe señalar un tipo de rima en el que se corta la palabra, se realiza una pequeña cesura para que la rima sea perceptible:

todo mi honor en tor/no a un tono que no sono (Ninguna chavala tiene dueño)

Genética y laringe, inge / niería letrística en el surco (Pura droga sin cortar)

presento la mentalidad sueka con beka

completa / mente loco por pecar (Ninguna chavala tiene dueño)

Se utiliza también otro tipo de rima en el que en vez de dividir una palabra se juntan dos que formarán una rima con otros vocablos. Veamos cómo en este fragmento rima "tú si", "sushi", "jacuzzi" y "puti", o en el siguiente fragmento "porno" y "favor no":

No nos culpes de errores que no hemos cometido y tú si,

Ahora ves el tuyo orgullo engullo crudo como el sushi,

Mc pussy puta cursi, no me hables de jacuzzi,

El único que has visto lo pagaste en un puti

asi las cosas, dedicate al porno,

(Pura droga sin cortar)

alli haz el ridiculo, en el rap ya, por favor no (Mierda)

Otro rasgo innovador consiste en la adición o supresión de un fonema o varios o letra para lograr una rima:

trae aquí ese líquido, sea lo que sea soy el don, ¿yo un ligón? non (Javat y Kamel)

Muchos amigos y pocos verdaderos, las borracheras no me ayudan a olvidar, non, ahora no puedo pero luego quiero veros, chicas podemos brindar en cuantos bares soy cliente de honor

(Ballantains) 
es el Jazz de yo y mi micrófono y lo toco yo

con otros dos, nosotros somos los gordos co (Atrás)

En los dos primeros casos utiliza el mismo término "non", en el primero para conseguir una rima consonante, en el segundo, sin embargo, parece un matiz estilístico, una llamada de atención al oyente, ya que la rima con "honor" en asonante estaba asegurada. En el tercer ejemplo encontramos la palabra "colega" reducida a "co", algo habitual en el argot de este grupo, y en su Zaragoza natal.

Como vemos, la libertad con la que se trata la lengua es total, la rima domina las letras y marca el ritmo. Los prejuicios que han dominado tradicionalmente entre los poetas y tratadistas de métrica acerca de la rima interna o del tan nombrado "porrazo del consonante" no influyen en este estilo, en el que las rimas se suceden sin miedo al ripio:

tengo fe en la fonética, me escuchas y me sientes, creo que no giramos en órbitas diferentes,

ya que son patentes las fuentes de amor que generé en

mis letras son puentes suficientes (Javat y Kamel)

las gentes,

La rima puede forzar el acento, como hemos visto, dividir sílabas, unir o separar palabras, e incluso se modifica la correcta gramática de la frase para lograrla, o se fuerzan estructuras:

Ahora ves el tuyo orgullo engullo crudo como el sushi

(Pura droga sin cortar)

Efectivamente, jodíase el que del rap mal hablase (Intro 97)

Queda, por último, comentar que, como se ha podido observar, tampoco Violadores del Verso evitan la rima con palabras extranjeras, ni vulgarismos, al contrario, la utilizan con mucha frecuencia y naturalidad:

¿Competición? Est non. El campeón dijo (Modestia aparte)

Que decías, a mi chaval me pagan los flows, 
Pero sin duda el mejor premio es el aplauso en los shows

(Pura droga sin cortar)

flipo con el ritmo y pienso bon voyage,

miro afuera y todas las luces van pa' trás (Pura droga sin cortar)

vengo montao en un Gran Jeep,

un bic, un block, un lápiz, un fat beat (Pura droga sin cortar)

Con esto cerramos el análisis de la rima en este grupo. Como se puede ver, hemos utilizado un corpus limitado de canciones, que ha sido suficiente para encontrar una gran cantidad de usos que podríamos llamar experimentales en la rima. El rap, que erige la rima como base fundamental de su ritmo, de su estilo, se configura como un campo de ensayo muy rico para este recurso.

\section{LA RIMA EXTRASISTEMÁTICA EN LA POESÍA}

La rima ha sido un elemento métrico discutido, ya que para algunos estudiosos (Esteban Torre, Rudolf Baehr, Tomás Navarro Tomás, Juan Frau) podría ser considerado no como un componente fundamental del verso, sino más bien un elemento dentro de un conjunto de recursos fónicos que se utilizan ocasionalmente en el verso y refuerzan el ritmo. Sin embargo, aunque el verso no necesita obligatoriamente la rima, ésta se nos presenta como un factor métrico fundamental por varias razones: su abundancia en la poesía en español hasta la época actual; ha estado tan presente tradicionalmente en la poesía en lengua española que un tratadista como Luis Mario llega a decir: "A la versificación castellana ha llegado a llamársela no solo silábica y acentual, sino también rímica, por el uso que hacen los poetas de la rima"22. Por otra parte es uno de los componentes métricos que más identifica al verso para muchos receptores ${ }^{23}$, ya que es fácilmente

22 MARIO, Luis: Ciencia y arte del verso castellano, cit., p. 143.

23 PARAÍSO, Isabel: La métrica española en su contexto románico. Madrid: Arco Libros, 2000, p. 57. 
perceptible, como atestigua Balbín: "Entre los factores rítmicos que se integran en el concierto estrófico, el más inmediato y perceptible es el ritmo de timbre"24. Más importante aún es el hecho de ir colocada normalmente a final de verso, marcando, junto con la pausa, el límite del mismo ${ }^{25}$. Justamente por este último motivo, se puede defender una función métrica de la rima, además de su carácter eufónico y semántico. Muy esclarecedoras son las palabras de Domínguez Caparrós al respecto, pronunciándose a favor de una inclusión de la rima como elemento métrico:

La rima, como se indicó en el formalismo ruso, se diferencia, en el conjunto de los hechos eufónicos (orquestación verbal, aliteraciones, etc.), por su función rítmica, en cuanto que es un factor del metro, y ayuda al oído a percibir la descomposición métrica de la lengua poéti$\mathrm{ca}$, y la correspondencia entre sus partes ${ }^{26}$.

Al comienzo hacíamos ya referencia a la amplia polémica que se ha desarrollado en el campo poético sobre la conveniencia o no del uso de la rima. A través del tiempo podemos observar diferentes actitudes ante este elemento, autores que la defienden o que la rechazan vivamente ${ }^{27}$. Sin embargo, en cierto punto la poesía, que se había mantenido a medio camino entre lo oral y lo escrito, prácticamente pierde este carácter hablado y pasa al dominio de la recepción como texto escrito. Esto sucede bastante recientemente, pues como dice Martín Casamitjana: "La decadencia de la rima se sentía como una novedad todavía en los inicios de los años treinta"28, y recoge

${ }^{24}$ BALBÍN, Rafael de: Sistema de rítmica castellana. Madrid: Gredos, 1968, p. 219.

${ }_{25}$ Lo señalan varios autores como: QUILIS, Antonio: Métrica española. Barcelona: Ariel, 1993, p. 84, y también DOMÍNGUEZ CAPARRÓS, José: Métrica española. Madrid: Síntesis, 2000, p. 124.

26 DOMÍNGUEZ CAPARRÓS, José: "La rima: entre el ritmo y la eufonía", cit., pp. 153.

27 Luis MARIO recoge testimonios de otros poetas que se han mostrado contrarios a la rima (aunque en la práctica muchos la hayan utilizado), desde Verlaine hasta Delmira Agustini, Manuel González Prada o Amado Nervo. (MARIO, Luis: Ciencia y arte del verso castellano, cit., pp. 140-142.)

28 MARTÍN CASAMITJANA, Rosa $\mathrm{M}^{\mathrm{a}}$ : El humor en la poesía española de vanguardia, cit., p. 58. 
un poema de Manuel Soriano de esa época titulado "La rima en crisis" que dice así:
Causa pena, causa duda
y a nadie le cabe duda
que hoy día sufre la rima
una crisis muy aguda

Un estudio de $\mathrm{Frau}^{29}$ sobre la rima en el verso español actual analiza un corpus de 1515 poemas de autores españoles aparecidos en antologías que recogen composiciones escritas entre 1975 y 2003 . Según los datos que recoge, de estos elementos solamente un $12 \%$ son rimados, y el $88 \%$ restantes carecen de rima. Así, vemos la tendencia que existe en la poesía española a la poesía sin rima. Muchos son los motivos de esta crisis. En primer lugar se le achaca el defecto de su excesiva sonoridad, por ello es más aceptada y utilizada actualmente la rima asonante, si excluimos los sonetos ${ }^{30}$. Por otra parte, la rima aparece ligada en la mentalidad de poetas y lectores a un cierto tipo de combinaciones métricas; esto es, no se toma como un elemento libre, que puede aparecer en cualquier tipo de versificación, sino que se liga quizás también a composiciones de estructura fija, como pueden ser el soneto, el romance, etc ${ }^{31}$. En la práctica poética advertimos, por ejemplo, que se suele oponer rima a verso libre ${ }^{32}$. Cierto es, por otra parte, que el verso libre ha prescindido en su práctica normalmente de una organización precisa, pero igual que podemos hablar del uso de metros tradicionales en verso libre, no sería descabellado hablar de rimas más o menos esporádicas en este tipo de versificación.

29 FRAU, Juan: "La rima en el verso español”. Rhythmica. Revista española de métrica comparada, 2004, II, pp. 109-136.

$30 \quad$ Ibid., p. 129.

31 FRAU llega a la siguiente conclusión en su estudio: "La rima pervive, sobre todo, en tres tipos de composición: por un lado, en el caso mencionado del soneto, estrofa predilecta para muchos poetas y lectores; por otra parte, como se ha visto, en estructuras arromanzadas y en estructuras donde la rima se distribuye de modo irregular". Ibid., p. 129.

32 Dentro de la versificación irregular destacan los poemas rimados de Rubén DARÍO como la "Salutación a Leonardo" que se trata de una versificación por clásulas rítmicas con rima entre los versos. Asimismo, poemas de Leopoldo LUGONES podrían considerarse también verso libre rimado. 
Sin embargo, la función de la rima en este tipo de composiciones sería, ya que no es sistemática y su distribución no es regular, meramente eufónica y no rítmica, como recoge Domínguez Caparrós en un estudio en el que analiza varios casos límite de rima: "Sea suficiente con los analizados para pensar que quizá la rima, en la época del verso amétrico, no tiene más función que la meramente eufónica, de leve contraste entre sonidos que se repiten y los que no, para producir la sensación del tiempo sobre el recuerdo" ${ }^{33}$. En todo caso, la abundancia del verso libre en la versificación española contemporánea ha sido un factor clave en la decadencia de la rima. Afirma Frau: "El triunfo de unas formas conlleva que otras queden relegadas a un segundo plano, y podemos suponer que el hecho de que las estrofas clásicas se utilicen, relativamente, en raras ocasiones - con la notable excepción, ya reseñada, del soneto-, lleva aparejado el consiguiente descenso en el uso de la rima" 34 .

¿Queda entonces campo de experimentación para un elemento tan ligado a la tradición? La poesía, desde el siglo de Oro a la actualidad ha jugado también a experimentar métricamente con todos sus elementos, entre ellos la rima. Por ello no nos es ajeno el concepto de tmesis o encabalgamiento léxico, que colabora con la rima gran parte de las veces. Este recurso no es nuevo para la poesía, y ha sido utilizado desde antiguo en nuestra literatura, quizás por imitación de la poesía clásica grecolatina. Veamos uno de los ejemplos más conocidos:

\section{Y mientras miserable- mente se están los otros abrazando con sed insacïable}

(Fray Luis de León, “A la vida solitaria”)

\footnotetext{
33 DOMÍNGUEZ CAPARRÓS, José: "La rima: entre el ritmo y la eufonía", cit., p. 169.

34 FRAU, Juan: "La rima en el verso español", cit., p. 128.
} 
Ya entonces tenía sus detractores ${ }^{35}$, como Quevedo ${ }^{36}$, y ha sido un recurso muy criticado y poco utilizado hasta el siglo Xx. Los casos más conocidos son los que separan en dos partes los adverbios terminados en -mente, como el que hemos visto de Fray Luis de León o casos en los que se divide una palabra compuesta. Estos tipos son más aceptados pues parece existir una doble acentuación en la palabra. Poetas del siglo Xx han hecho uso igualmente de este recurso:

El alma que se advierte sencilla y mira claramente la gracia pura de la luz cara a cara

(Darío, XV, Cantos de vida y esperanza)

Porque escribir es viento fugitivo, y publicar, columna arrinconada. Digo vivir, vivir a pulso, airadamente morir, citar desde el estribo

(Blas de Otero, "Digo vivir")

...dulcísimo, le asestas una espada

-siete en el naipe, muchas al tableroy lo más delicadamente posible se abre un agujero...

(Carvajal, "Siete de espadas")

Lo que resulta más extraño es la utilización del encabalgamiento léxico (con el fin de la rima) con otro tipo de palabras, aunque no faltan ejemplos:

¡Oh, ciego!, ; oh, sordo!

¡oh, mudo! Yo

te daba opio,

de daba bro-

muro, té, método

(J. R. Jiménez, "Lamento de primavera")

35 OROZ ARIZCUREN estudia las críticas a Fray Luis por la utilización de la tmesis. (OROZ ARIZCUREN, Francisco J.: "Una licencia poética de Fray Luis Miserable-Mente criticada”. Iberomania, 1984, 20, pp. 57-74.) Anteriormente había sido ya estudiado por QUILIS, Antonio: "Los encabalgamientos léxicos en -mente de fray Luis de León y sus comentaristas". Hispanic Review, 1963, 31, pp. 22-39.

36 RIVERS, Elias L.: "Preceptismo dogmático de Quevedo: su condena del encabalgamiento léxico y del hipérbaton”. La Perinola: revista de investigación quevediana, 2001, 5, pp. 277-284. 
Tiempo de soledad es éste. Suena en Europa el tambor de proa a popa. Ponte la muerte por los hombros. Ven. Alejémonos de Europa

(Blas de Otero, "Paso a paso")

Vemos la tmesis empleada de dos maneras diferentes. En el caso de Juan Ramón Jiménez la palabra, al partirse, crea un acento en la última sílaba del verso "bro", que produce la rima. En Blas de Otero, sin embargo, no se crea un nuevo acento, sino que se unen dos palabras y rima "suena" con "ven.-A". Es digno de resaltar, igualmente, que las palabras no se rompen después de la última sílaba acentuada, que parecería lo más común, sino en otro punto que el poeta ha considerado más adecuado.

La rima que se produce gracias a la tmesis se ha venido utilizando en la tradición poética de nuestro siglo, casi siempre unida a juegos de ingenio, humor, aunque a veces también como recurso expresivo cargado de significado. Antonio Carvajal es uno de los poetas que más interés ha mostrado en este tipo de experimentación, y ha acuñado incluso el término de "versos de cabo doblado"37 por contraposición a los de "cabo roto" cervantinos y que explica así: "Doblar el cabo del verso, pasar su sílaba o sílabas postónicas" 38 . Veamos un ejemplo:

Oh nube, cuánta caléndula en flor espera lluvia que le niegas tan huraña y avara sabiendo que es el agua sostén de la flor y la alegría de cuerpos y alma ardidos! ¡No celes sol, y colma la sed con unos gol-

37 DOMÍNGUEZ CAPARRÓS dedica un artículo a este fenómeno en la poesía de Antonio CARVAJAL. (DOMÍNGUEZ CAPARRÓS, José: "Versos de cabo doblado", en Nuevos estudios de métrica. Madrid: Universidad Nacional de Educación a Distancia, 2007, pp. 83-89.)

38 CARVAJAL, Antonio: Poética y poesía. Edición de Antonio Gallego. Madrid: Fundación Juan March, 2004, p. 31. 
pes generosos y fluidos de ti!

(Carvajal, “Ad petendam pluviam”)

Es obvio que el sentido de estos encabalgamientos y sus rimas es simplemente el juego con el sonido, la experimentación. Cabe comentar que entre el tercer y el cuarto verso encontramos la separación de "hu / raña", en la que no se pasa la sílaba postónica al siguiente verso, sino que se acentúa la sílaba "hu", que sería átona en su contexto normal, y se pasa al siguiente verso la sílaba tónica y la postónica "raña". Esto en ocasiones persigue incluso rimar palabras fénix o sin rima consonante posible:

...del trueno y el relámpago

del existir, vorágine de besos, irresistible trampa, gozoso esplendor de ilesos ángeles, que no humanos, inconfesos...

(Carvajal, "Vista de Badajoz al atardecer")

Algo que puede resultar aún más violento es la división incluso de sílabas, como sucede en estos versos:

...la vida con gozo!

Bajo del conde-

stable cielo, ciegos del reverbero

de su gloria, mientras se esconde

el orgullo, que artero...

(Carvajal, "Piedra viva")

Lo cierto es que es un caso particular en el que no se pretende simplemente romper una sílaba sino, como apunta el propio autor, "jugar con la doble etimología, la culta [comes stabuli] y la popular, y enriquecer el texto con semas imprevistos" ${ }^{\prime 39}$.

Dignos de comentario son otros casos de experimentación que ya habíamos comentado a partir de las letras de Violadores del Verso, que se basan en la consecución de la rima 39 Ibid., p. 29. 
mediante la ensayo y cambio, de varios tipos, de los acentos de la palabra. Uno de estas experimentaciones parte del ya mentado Antonio Carvajal, y es la llamada "rima en caída", que Domínguez Caparrós define de la siguiente manera:

El fenómeno ha sido bautizado así por Antonio Carvajal, y consiste en disociar la rima del acento, para considerar con plena entidad fónica a efectos de la rima todos los sonidos que siguen a la vocal acentuada de una palabra. El resultado son rimas como las siguientes: ópalo / desamparo, asonante en ao; o consonancias en ima entre rima, óptima, última; y en alos entre ópalos, sándalos, halos ${ }^{40}$.

Lo que puede parecer un hallazgo del poeta granadino se ejemplifica con versos de García Lorca, Cernuda, Altolaguirre, Carlos Pellicer y Alfonsina Storni. Esto demuestra que, consciente o inconscientemente, este tipo de rima es perceptible, tiene sonoridad. Sin embargo, es un recurso que no se utiliza de manera consciente y constante en nuestro país hasta Antonio Carvajal. Veamos algún ejemplo del poeta granadino:

Generosas de pétalos, de olores son algo avaras: Tilos y petunias se dan enteros; mas las alegrías y las celindas y las rosas, pobres

(Carvajal, "Flores del norte")

Esta rima es muy llamativa y complicada, pues en ella debe rimar un hiato con un diptongo no acentuado.

Hemos dicho que Carvajal es quizás el primer poeta español que utiliza este recurso deliberadamente y le da nombre. Sin embargo, en Latinoamérica otro poeta y estudioso de la versificación cuantitativa había llegado ya a un concepto semejante, que ya hemos nombrado en este trabajo: se trata de la rima potencial de Luis Ángel Casas. Recordemos que se trataba de un tipo de rima que se basa en "la igualdad silábica pura o semejanza silábica pura, según los casos, pero siempre

40 DOMÍNGUEZ CAPARRÓS, José: "La rima: entre el ritmo y la eufonía”, cit., p. 160. 
con independencia del acento"41. Es fácil apreciar el parecido entre ambos conceptos. La llamada "rima potencial" es, no obstante, un concepto mucho más amplio, en el que caben rimas como "labios" con "viola" por inversión de sílabas o "tiempo" y "contemplaba" que establecen semejanza tónica por las dos primeras sílabas de la última palabra. Lo que quiero hacer notar es que en la rima en caída los sonidos que cuentan son únicamente aquellos postónicos, mientras que en la rima potencial cabe tener en cuenta los anteriores al acento tónico de la palabra, como sucedía en la rima de colega, cólera, dolerá, bóveda y moverá, que veíamos en la letra de Violadores del Verso.

Tras esto sobra decir que no toda la poesía ni todos los autores han rechazado la rima a lo largo del tiempo. Encontramos casos de experimentación desde las primeras épocas de este género en nuestro idioma, y ese afán de renovación ha continuado en algunos casos hasta nuestra época. No obstante, hay diferencias en el uso de estas innovaciones en la rima entre dos géneros que pueden resultarnos tan distantes como el hip-hop y la poesía, y que, sin embargo, han demostrado tener mucho en común.

\section{Conclusiones}

Después de realizar este trabajo creo que es destacable, en primer lugar, la gran cantidad de poetas y poemas en los que se ha experimentado con la rima. Esta experimentación, como hemos visto, no comienza en nuestra época, sino que se remonta a los comienzos de nuestra tradición poética.

Según lo visto, la rima, al igual que otros elementos métricos, ha sido ampliamente tratada en los estudios literarios desde el Renacimiento. Gracias a ello contamos con testimonios de tipos de rimas utilizados y de su consideración por parte de poetas renombrados y tratadistas. Si examinamos las innovaciones rímicas que hemos ido observando en las letras de Violadores del Verso y en las composiciones de poetas contempo-

$\overline{41}$ MARIO, Luis: Ciencia y arte del verso castellano, cit., p. 151. 
ráneos, daremos en la cuenta de que casi sin excepción todos ellos habían ya sido utilizados en la tradición poética, si bien siempre no como algo establecido sino solamente experimental. La rima por tmesis es quizás la que ha contado con un cultivo más continuado (nunca exento de polémica), siendo utilizada con gran ingenio $\mathrm{y}$, en muchos casos, con matiz humorístico:
En Cacabelos un chulo acaba de descubrir la cuadratura del cir- culo.
En vano la envidia ladra, el gran Novoa ;oh ventura! ha descubierto la cuadra- tura.
Denle al momento una placa que bien la merece ¡cielos! el geómetra de Caca- belos ${ }^{42}$.

Pero no faltan ejemplos de rima por cambio de acento, con extranjerismos o vulgarismos, por invención de palabras, o para forzar la rima de una palabra fénix. Veamos este ejemplo de Quevedo, en el que vemos varias de estas características:
Al escribir con mi lápiz he cometido un desliz. Resulta que he escrito tápiz, en vez de escribir tapiz ${ }^{43}$.

Incluso la tan temida y criticada rima interna ha sido utilizada por algunos de los más grandes poetas del siglo Xx:

Una querencia tengo por tu acento, una apetencia por tu compañía y una dolencia de melancolía por la ausencia del aire de tu viento.

(Miguel Hernández, "Una querencia tengo por tu acento")

\footnotetext{
42 VALERA, Juan: Correspondencia. Barcelona: Linkgua Ediciones, 2007.

43 Extraído de GARMENDIA GIL, José Antonio: Florilegio de chorradas. Signatura Ediciones, Sevilla, 2000, p. 1.
} 
De "rima en caída", contamos con ejemplos encontrados por el propio Antonio Carvajal en García Lorca, Cernuda y otros poetas del siglo Xx. Veamos el poema "Arbolé, arbolé" de Lorca en el que encontramos una rima por cambio del acento de una sílaba a otra y una rima en caída:

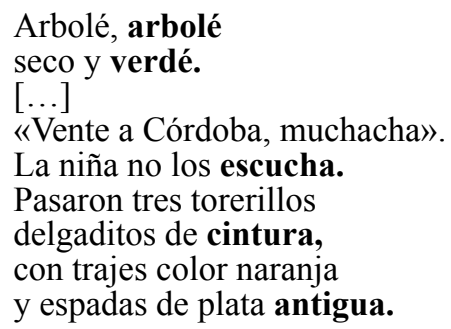

(Federico García Lorca, “Arbolé, arbolé”)

Sin embargo, toda esta producción y experimentación con la rima ha sido puntual, es decir, utilizada como una excepción dentro de la obra de un autor, bien sea como juego o incluso de manera no deliberada (como parece que es en el ejemplo de Lorca). Solamente ciertos poetas, como Antonio Carvajal o el cubano Luis Ángel Casas han realizado una apuesta fuerte y consciente por estos nuevos tipos de rima, que de no entrar en funcionamiento por otros poetas quedarán como empezaron, como un simple experimento. Preclaras son las palabras al respecto de Luis Mario respecto a la rima potencial:

Nadie puede afirmar o negar si tendrá alguna acogida en la poética del futuro, aunque para ello tendrían los poetas que volver a la mesa de estudio. Ello no parece probable por ahora, en un mundo dado cada vez más al tecnicismo, excepto en el arte, que es la gran víctima, el gran burlado por la improvisación y la irrespetuosidad de los últimos tiempos ${ }^{44}$.

Por ello ciertos estilos musicales ofrecen a la rima un campo en el que, a diferencia de la poesía, sí cabe una estandarización y una utilización no marcada de estos fenómenos que en poesía parecen menos aceptados al relacionarse mayorita-

$\overline{44}$ MARIO, Luis: Ciencia y arte del verso castellano, cit., p. 151. 
riamente con asuntos humorísticos o simplemente a la muestra de ingenio.

La conclusión fundamental de este trabajo es que, si bien en la tradición poética ha existido experimentación en los límites de la rima, esta nunca, o casi nunca, ha sido un intento de renovación real, sino más bien tiende a tener un carácter de juego de ingenio puntual. Solamente algunos poetas han hecho intentos reales de introducir estas rimas que podríamos llamar periféricas en algo permanente en su poesía -nos referimos a Luis Ángel Casas y Antonio Carvajal-. La aportación del hip-hop, en este caso de Violadores del Verso, es que se convierten estas innovaciones, esta rima extrasistemática en algo básico para la letra-poema, en un elemento organizativo fundamental, es decir, en algo sistemático.

En todo caso este estudio pretende dar cuenta de la fuerte ligazón que existe entre los recursos utilizados en la poesía y las letras de algunos géneros musicales, cuyo estudio ha sido muy limitado en el ámbito académico y que, sin embargo, aportan un aire nuevo en muchos aspectos. Según hemos visto, la rima es un elemento fundamental para el rap, y en sus letras podemos encontrar todo tipo de novedades utilizadas sin ningún prejuicio. Así, aunque la poesía del siglo xx ha experimentado en cierta medida con la rima, parece que los poetas han sentido hacia ella algo de recelo, sobre todo en su uso abusivo, por el temor de caer en el vicio de la sonoridad y olvidar el sentido. En relación a la rima en la vanguardia apuntaba Rosa $\mathrm{M}^{\mathrm{a}}$ Martín Casamitjana: "Al relacionarse las palabras por su sustancia fónica, se produce una hipertrofia de un aspecto secundario del lenguaje en detrimento de su aspecto primario, el significado" 45 . Las letras musicales, al no tener el carácter "sagrado", serio y culto que rodea a la poesía, se arriesgan en la utilización de la rima, sin recelos hacia su sonoridad, es más, explotándola, otorgándole valores semánticos nuevos. Por ello, el estudio comparado de la utilización de la rima en diversos géneros y estilos puede resultar muy enriquecedor para cualquiera de ellos.

45 MARTÍN CASAMITJANA, Rosa Mª El humor en la poesía española de vanguardia, cit., p. 66. 


\section{Bibliografía}

BALBÍN, Rafael de: Sistema de ritmica castellana. Madrid: Gredos, 1968.

CARVAJAL, Antonio: Poética y poesía. Edición de Antonio Gallego. Madrid: Fundación Juan March, 2004.

DEVOTO, Daniel: Para un vocabulario de la rima española. Paris: Klincksieck, 1995.

Diccionario de la lengua española. $\mathrm{http} / / /$ buscon.rae.es/draeI/html/cabecera.htm, 2001.

DOMÍNGUEZ CAPARRÓS, José: "La rima: entre el ritmo y la eufonía", en Estudios de métrica. Madrid: Universidad Nacional de Educación a Distancia, 1999, pp. 149-169.

- Métrica española. Madrid: Síntesis, 2000.

_ "Versos de cabo doblado", en Nuevos estudios de métrica. Madrid: Universidad Nacional de Educación a Distancia, 2007, pp. 83-89.

FRAU, Juan: "La rima en el verso español”. Rhythmica. Revista española de métrica comparada, 2004, II, pp. 109-136.

— "Teorías y polémicas sobre la rima en el Renacimiento inglés". Rhythmica. Revista española de métrica comparada, 2008, V-VI, pp. 49-90.

GARMENDIA GIL, José Antonio: Florilegio de chorradas. Signatura Ediciones, Sevilla, 2000.

GÜELL, Mónica: "La creatividad de las rimas en las décimas y letrillas de Góngora", en Anthony J. Close y Sandra María Fernández Vales (coords.), Edad de oro cantabrigense. Actas del VII Congreso de la Asociación Internacional de Hispanistas del Siglo de Oro. Asociación Internacional del Siglo de Oro, 2006, pp. 331-337.

MARIO, Luis: Ciencia y arte del verso castellano. Miami: Ediciones Universal., 1991.

MARTÍN CASAMITJANA, Rosa $\mathrm{M}^{\mathrm{a}}$ : El humor en la poesía española de vanguardia. Madrid: Gredos, 1996.

MARTÍNEZ FERNÁNDEZ, José Enrique: El fragmentarismo poético contemporáneo. León: Universidad de León, 1996.

OROZ ARIZCUREN, Francisco J.: "Una licencia poética de Fray Luis Miserable-Mente criticada". Iberomania, 1984, 20, pp. 57-74.

PARAÍSO, Isabel: La métrica española en su contexto románico. Madrid: Arco Libros, 2000.

PUJANTE CASCALES, Basilio: "La retórica del rap. Análisis de las figuras retóricas en las letras de Violadores del Verso". Tonos digital: Revista electrónica de estudios filológicos, 2009, 17.

QUILIS, Antonio: "Los encabalgamientos léxicos en -mente de fray Luis de León y sus comentaristas". Hispanic Review, 1963, 31, pp. 22-39. - Métrica española. Barcelona: Ariel, 1993.

RIVERS, Elias L.: "Preceptismo dogmático de Quevedo: su condena del encabalgamiento léxico y del hipérbaton". La Perinola: revista de investigación quevediana, 2001, 5, pp. 277-284.

SANTOS UNAMUNO, Enrique: "El resurgir de la rima: los poetas románicos del rap", en Antonella Cancellier, Renata Londero (coords.), Atti del XIX Convegno [Associazione ispanisti italiani]: Roma, 16-18 settembre 1999, vol. 2, 2001 (Italiano e spagnolo a contatto), pp. 235-242.

SUTIL, Miguel Ángel: Los hijos secretos del funk. Zaragoza: Zona de obras, 2008.

VALERA, Juan: Correspondencia. Barcelona: Linkgua Ediciones, 2007. 